\title{
Influência das Atividades de Autocuidado de Pacientes Diabéticos Sobre os Parâmetros Bioquímicos e Antropométricos
}

\author{
Raiza Lima do Carmo ${ }^{1}$, Tatiane Dutra Nunes ${ }^{2}$, \\ Tatiele Dutra Nunes ${ }^{3}$, Karine Santos de Bona Libardoni ${ }^{4}$
}

\begin{abstract}
RESUMO
Objetivo: Avaliar a influência de atividades de autocuidado por pacientes com Diabetes Mellitus sobre parâmetros bioquímicos e antropométricos. Materiais e métodos: Estudo observacional transversal, desenvolvido com pacientes diabéticos atendidos pelo Sistema Único de Saúde na cidade de Santo Ângelo-RS. O instrumento de pesquisa utilizado para avaliar o autocuidado foi um Questionário de Atividades de Autocuidado com Diabetes. Foram feitas coletas de sangue para determinação de parâmetros bioquímicos, como glicose, colesterol total, lipoproteína de alta e baixa densidade, além da determinação de parâmetros antropométricos, como o Índice de Massa Corporal e medidas da circunferência abdominal e do quadril para cálculo de relação circunferência-quadril. Para análise dos dados foi utilizado Teste $t$ de Student, sendo considerados significativos valores com $p$ $<0,05$ Resultados: A amostra foi composta por 65 pacientes que possuíam Diabetes tipo 2 ( $52 \%$ eram homens e $48 \%$ mulheres), com idade média de $60 \pm 11$; Destes, $60 \%$ possuem Ensino fundamental incompleto. A alta adesão ao autocuidado (grupo 1 ) foi evidenciada em $78 \%$ dos pacientes e $22 \%$ enquadraram-se com baixa adesão ao autocuidado (grupo 2). 0 grupo 2 apresentou valores significativamente mais elevados nos parâmetros circunferência abdominal, de quadril e a relação entre as duas, assim como nos níveis de colesterol total e triglicerídeos. Ainda, 39\% dos pacientes do grupo 1 e $57 \%$ dos pacientes do grupo 2 apresentaram-se obesos. Conclusão: $\mathrm{O}$ autocuidado pode influenciar nos parâmetros bioquímicos e antropométricos dos pacientes diabéticos, com as alterações sendo observadas de acordo com o grau de autocuidado dos pacientes.
\end{abstract}

Palavras-chave: Autocuidado. Diabetes mellitus. Obesidade. Sistema Único de Saúde.

\section{INFLUENCE OF SELF-CARE ACTIVITIES OF DIABETIC PATIENTS ON} BIOCHEMICAL AND ANTHROPOMETRIC PARAMETERS

\begin{abstract}
Objective: To evaluate the influence of self - care activities by patients with Diabetes mellitus on biochemical and anthropometric parameters. Materials and methods: Cross-sectional observational study developed with diabetic patients attended by the Unified Health System in the city of Santo Ângelo-RS. The research tool used to assess self-care was a Diabetes Self-Care Activities Questionnaire. Blood samples were collected for the determination of biochemical parameters, such as glucose, total cholesterol, high and low density lipoprotein, as well as determination of anthropometric parameters, such as Body Mass Index and abdominal and hip circumference measurements for circumference hip. For the analysis of the data, Student's t-test was used, being considered significant values with $p<0.05$. Results: The sample consisted of 65 patients who had type 2 diabetes (52\% were men and $48 \%$ women), with a mean age of $60 \pm 11$, of which $60 \%$ have incomplete elementary education. A high adherence to self-care (group 1) was observed in $78 \%$ of the patients and $22 \%$ of the patients were classified with low adherence to self-care (group 2). Group 2 presented significantly higher values in the waist circumference, hip ratio and the relationship between the two, as well as total cholesterol and triglyceride levels. Still, $39 \%$ of patients in group 1 and $57 \%$ of patients in group 2 were obese. Conclusion: Self-care can influence the biochemical and anthropometric parameters of diabetic patients, and the changes are observed according to the degree of self-care of the patients.
\end{abstract}

Keywords: Self-care. Diabetes mellitus. Obesity. Health Unic System.

Recebido em: $17 / 7 / 2017$

Avaliações requeridas em: 20/9/2018

Aceito em: 9/11/2018

\footnotetext{
Mestranda do Programa de Pós-Graduação em Medicina: Ciências Médicas - UFRGS. Graduação em Farmácia pela Universidade Regional Integrada do Alto Uruguai e das Missões (2017). raizadocarmo@hotmail.com

2 Acadêmica do Curso de Farmácia da Universidade Regional Integrada do Alto Uruguai e das Missões - URI Santo Ângelo.

Acadêmica do Curso de Farmácia da Universidade Regional Integrada do Alto Uruguai e das Missões - URI Santo Ângelo.

${ }^{4}$ Doutora em Ciências Farmacêuticas com ênfase em Farmacologia pela Universidade Federal de Santa Maria e Professora do Curso de Farmácia da Universidade Regional Integrado do Alto Uruguai e das Missões - URI Campus Santo Ângelo.
} 


\section{INTRODUÇÃO}

O Diabetes mellitus (DM) é caracterizado por uma disfunção no pâncreas, o órgão produtor de insulina, hormônio responsável pela monitorização dos níveis de glicose no organismo, e geralmente é um resultado de defeitos na secreção e/ou ação da insulina (SANTOS et al., 2014).

O DM tipo 2 possui forte influência da herança genética, que ainda não está completamente esclarecida, fatores ambientais, como hábitos alimentares e inatividade física, todos fortes contribuidores para o desencadeamento da doença. A persistente elevação da glicemia está associada a complicações micro e macrovasculares, diminuição da qualidade de vida e aumento da mortalidade de pacientes (OLIVEIRA; MONTENEGRO JUNIOR; VENCIO, 2017).

O número de pessoas com a doença vem crescendo em todos os países do mundo. No ano de 2015 estimava-se que $8,8 \%$ da população mundial tivesse DM. No mesmo ano, o Brasil ocupava o quarto lugar no ranking de países com maior número de diabéticos, possuindo 14,3 milhões de pessoas com a doença (OLIVEIRA; MONTENEGRO JUNIOR; VENCIO, 2017).

O diagnóstico na maioria das vezes é tardio, pois muitos pacientes acabam não dando atenção aos sinais clínicos da doença e a descobrem por acaso em exames de rotina. Por esse motivo, salienta-se a importância da prevenção primária para a doença, principalmente para pessoas que possuem casos de DM na família e/ou apresentem algum risco de adquiri-la (OLIVEIRA; MONTENEGRO JUNIOR; VENCIO, 2017).

O DM é classificado como uma doença crônica, e o principal objetivo do tratamento é a manutenção da glicemia em seus níveis próximo ao normal. Além disso, tenta-se regular o metabolismo da insulina, evitar o aparecimento de outras doenças, diminuir transtornos alimentares, bem como o estímulo para uma vida mais saudável (BRASIL, 2013).

Para uma escolha do tratamento adequado é necessário levar em consideração o estado geral do paciente, as comorbidades presentes, os valores de glicemia de jejum e pós-prandial, valores de hemoglobina glicada, peso, idade e possíveis interações que possam ocorrer com outros medicamentos já utilizados pelo paciente, bem como efeitos adversos e contraindicações (OLIVEIRA; MONTENEGRO JUNIOR; VENCIO, 2017).

Como primeira opção está o tratamento não medicamentoso, uma vez que a mudança de hábitos colabora para a melhoria do controle metabólico, reduz a necessidade da utilização de antidiabéticos orais ou injetáveis, contribui e estimula o emagrecimento nos pacientes obesos, diminui os riscos de doenças cardiovasculares e melhora a qualidade de vida, ressaltando-se que o incentivo para estas mudanças é fundamental (BRASIL, 2013). Por esse motivo, é importante a conscientização dos diabéticos para que desenvolvam habilidades para seu próprio cuidado, mostrando-lhes os benefícios desta prática e também os malefícios quando estes cuidados não são adotados. Esta prática é conhecida como autocuidado.

O autocuidado é definido como hábitos que a pessoa pode adotar para seu próprio benefício para melhor manutenção de sua vida, saúde e bem-estar. $O$ autocuidado envolve o seguimento de um plano alimentar, monitorização da glicemia capilar, realização de atividade física, uso correto dos medicamentos e cuidados com os pés, entre outros, visando assim a diminuir as comorbidades causadas pelo DM (GOMIDES et al., 2013).

Neste contexto, é preciso que o paciente desenvolva consciência de que o tratamento não medicamentoso, como alimentação balanceada, prática de atividades físicas, peso corporal saudável e abandono de práticas nocivas contribuem para uma melhor qualidade de vida e são essenciais na redução das complicações da doença, bem como para a sua prevenção. Dessa forma, em razão da importância do autocuidado para o tratamento e a prevenção das complicações crônicas do DM, o presente trabalho teve como objetivo avaliar a influência do autocuidado nos parâmetros bioquímicos e antropométricos de pacientes diabéticos atendidos pelo Sistema Único de Saúde do município de Santo Ângelo, RS.

\section{MÉTODOS}

O presente estudo foi aprovado pelo Comitê de Ética da Universidade Regional Integrada do Alto Uruguai e das Missões - URI campus Santo Ângelo por meio do CAAE no 62161616.8.0000.5354. Trata-se de uma pesquisa transversal analítica.

O estudo foi desenvolvido com pacientes cadastrados na lista de diabéticos do Posto de Saúde Doutor Ernesto Nascimento Sobrinho, do município de Santo Ângelo. Todos os pacientes que constavam nesta lista foram convidados a participar da pesquisa, porém aceitaram apenas os que tinham interesse no estudo e disponibilidades. Sendo assim, o estudo contou com 65 pacientes que atenderam aos critérios estabelecidos e concordaram com o Termo de Consentimento Livre e Esclarecido (TCLE). O estudo desenvolveu-se no período de março a junho de 2017. Os critérios 
de inclusão na amostra foram: pacientes diabéticos maiores de 18 anos cadastrados no SUS, que comparecerem na Universidade em jejum de no mínimo 8 horas, sem restrição de sexo, classe social ou cor da pele. Os critérios de exclusão foram: pacientes com problemas de alcoolismo, com presença de neoplasias em tratamento quimioterápico, assim como também gestantes.

Para coleta dos dados inicialmente os pacientes diabéticos que constavam na lista do posto de saúde foram convidados a participar da pesquisa, e com aqueles que concordaram em participar foram agendados encontros. Durante estes momentos foram coletados dados sociodemográficos por meio de entrevista individual com os pacientes. Aos mesmos pacientes foi aplicado um Questionário de Atividades de Autocuidado com o Diabetes (QAD), versão traduzida e adaptada para a cultura brasileira a partir do The Summary of Diabetes Self CareActivitiesMeasure (SDCA) (MICHELS et al., 2010). Neste questionário foram avaliadas informações como a alimentação geral e específica, atividade física, monitorização da glicemia, cuidado com os pés, uso de medicação e utilização de tabaco. Foram atribuídos valores para as respostas ao QAD de acordo com a frequência com que o paciente realizava a prática do autocuidado, conforme os dias da semana, destacando-se que para cada item os escores variam de zero a sete. O valor zero corresponde à situação menos favorável e o sete para a mais favorável. Nos itens relacionados com a alimentação específica que questionassem sobre o consumo de alimentos ricos em gordura e doces os valores foram invertidos, ou seja, zero é o mais favorável e sete o menos favorável.

Os pacientes foram divididos em dois grupos, de acordo com a pontuação obtida a partir do questionário $Q A D$, sendo eles de alta e baixa adesão. $O$ grupo 1 inclui pacientes com alta adesão ao autocuidado, ou seja, aqueles que obtiveram no QAD um somatório de mais que 40 pontos, e no grupo 2 foram incluídos pacientes com baixa adesão ao autocuidado, cujo somatório foi menor ou igual a 39 pontos.

Para avaliar os parâmetros estatura e peso utilizou-se de estadiômetro da marca Welmy e balança digital da marca Camry modelo EB9013, para cálculo do Índice de Massa Corporal (IMC), realizado pela equação peso/altura² ${ }^{2}$. A classificação destes parâmetros foi realizada com base na Diretriz Brasileira de Obesidade (ASSOCIAÇÃO..., 2016).

Além disso, com a utilização de fita métrica estimou-se a circunferência abdominal e do quadril, considerando-se valores normais para a circunferên- cia abdominal medidas até $80 \mathrm{~cm}$ para mulheres e $94 \mathrm{~cm}$ para homens. Com estas variáveis calculou-se a relação cintura quadril (RCQ), um índice muito importante, pois é eficiente na identificação de pessoas com risco de doenças cardíacas (SANTOS, 2014). Para a classificação destes foram utilizados os pontos de corte recomendados pela Word Health Organization (1998).

Após os questionários e determinação das medidas antropométricas, foram coletadas amostras de sangue total para obtenção do soro para determinação dos níveis de glicose e perfil lipídico, incluindo colesterol total, lipoproteína de alta densidade (HDL) e triglicerídeos. Utilizando os valores dessas dosagens foram calculados os níveis de lipoproteína de baixa densidade (LDL) e lipoproteína de densidade muito baixa (VLDL). O soro foi obtido por centrifugação das amostras de sangue total, em centrífuga Excelsa II da marca Fanem ${ }^{\circ}$, modelo $206-\mathrm{R}$, e as amostras foram todas processadas no laboratório de Bioquímica da Universidade Regional Integrada do Alto Uruguai -URI Campus Santo Ângelo. Na sequência foram feitas as análises utilizando kits comerciais da marca Laborclin', linha Bioliquid, conforme orientações descritas na bula de cada kit específico de dosagem. Os valores de referências utilizados para os parâmetros bioquímicos foram os estipulados pelas Diretrizes da Sociedade Brasileira de Diabetes (2017-2018) (OLIVEIRA; MONTENEGRO JUNIOR; VENCIO, 2017).

Para avaliar comparativamente os resultados obtidos, como dados antropométricos e bioquímicos, entre os grupos de alta e baixa adesão ao autocuidado foi aplicado o Teste t de Student, normatizado por meio do teste de Kolmogorov-Smirnov, com a utilização do programa GraphpadPrism e em todos os casos foi adotado nível de significância de $p<0,05$. As variáveis contínuas foram descritas por média e desvio padrão (DP) e as variáveis categóricas de acordo com a frequência.

\section{RESULTADOS}

A amostra estudada foi constituída por 65 pacientes que possuíam diagnóstico prévio de DM do tipo 2 (52\% homens e $48 \%$ mulheres), com idade média de $60 \pm 11,4$. O autocuidado dos pacientes foi identificado pelas respostas do QAD (Tabela 1), as quais possibilitaram dividir a amostra nos grupos de alta e baixa adesão ao autocuidado. 
Tabela 1 - Distribuição dos pacientes conforme a atividade de autocuidado com o Diabetes, levando-se em conta a média de dias (0-3 ou 4-7 dias) em que as atividades foram executadas na semana anterior à aplicação do QAD

\begin{tabular}{|c|c|c|}
\hline $\begin{array}{r}\text { Questões do Questionário de autocuida- } \\
\text { do com o Diabetes }\end{array}$ & 0-3 dias & 4-7 dias \\
\hline Seguiu uma dieta saudável? & 40 & 25 \\
\hline $\begin{array}{l}\text { Seguiu orientação alimentar dada por um } \\
\text { profissional de saúde (médico, enfermei- } \\
\text { ro, nutricionista)? }\end{array}$ & 53 & 12 \\
\hline $\begin{array}{l}\text { Comeu cinco ou mais porções de frutas e/ } \\
\text { ou vegetais? }\end{array}$ & 21 & 44 \\
\hline $\begin{array}{l}\text { Comeu alimentos ricos em gordura, como } \\
\text { carne vermelha e derivados de leite? }\end{array}$ & 49 & 16 \\
\hline Comeu doce? & 11 & 54 \\
\hline $\begin{array}{l}\text { Realizou atividade física durante pelo me- } \\
\text { nos } 30 \text { minutos (minutos totais de ativida- } \\
\text { de contínua, inclusive andar)? }\end{array}$ & 35 & 30 \\
\hline $\begin{array}{l}\text { Praticou algum tipo de exercício físico es- } \\
\text { pecífico (nadar, bicicleta, caminhar) sem } \\
\text { incluir atividades em casa ou no trabalho? }\end{array}$ & 59 & 6 \\
\hline Avaliou os níveis de açúcar no sangue? & 59 & 6 \\
\hline Examinou os seus pés? & 21 & 44 \\
\hline $\begin{array}{l}\text { Examinou dentro dos sapatos antes de cal- } \\
\text { çá-los? }\end{array}$ & 13 & 52 \\
\hline $\begin{array}{l}\text { Secou os espaços entre os dedos dos pés } \\
\text { depois de lavá-los? }\end{array}$ & 4 & 61 \\
\hline $\begin{array}{l}\text { Tomou o medicamento para diabetes con- } \\
\text { forme foi recomendado? }\end{array}$ & 9 & 56 \\
\hline $\begin{array}{l}\text { Você tem o hábito de fumar? Se sim, quan- } \\
\text { do consumiu o último cigarro? }\end{array}$ & 10 & 55 \\
\hline
\end{tabular}

Ao fazer a divisão dos grupos após a realização do questionário, $78 \%$ dos pacientes foram classificados com alta adesão ao autocuidado, os quais obtiveram 40 ou mais pontos com suas respostas no QAD. O grupo de baixa adesão foi composto por $21 \%$ dos pacientes.

Conforme a Tabela 2, de todos os pacientes estudados, 60\% possuem Ensino Fundamental incompleto, seguido de Ensino Médio completo (14\%). Ambos os grupos apresentaram semelhanças quanto à escolaridade, com prevalência de Ensino Fundamental incompleto. Todos os pacientes estudados são diagnosticados como diabéticos Tipo 2, apresentando tempo médio de diagnóstico da doença de aproximadamente $8( \pm 7)$ anos.
Tabela 2 - Caracterização dos participantes com DM, Santo Ângelo, RS, Brasil, 2017 ( $n=65$ )

\begin{tabular}{|l|c|c|c|}
\hline Variável & $\begin{array}{c}\text { Total } \\
(\mathrm{n}=65)\end{array}$ & $\begin{array}{c}\text { Grupo 1 } \\
\text { Alta } \\
\text { adesão } \\
(\mathrm{n}=51)\end{array}$ & $\begin{array}{c}\text { Grupo 2 } \\
\text { Baixa } \\
\text { adesão } \\
(\mathrm{n}=14)\end{array}$ \\
\hline Idade & $60 \pm$ & $58,84 \pm$ & $64,07 \pm$ \\
$11,53^{*}$ & 10,26 \\
\hline Gênero (F/M) & $31 / 34$ & $25 / 26$ & $6 / 8$ \\
\hline Escolaridade & 6 & 5 & 1 \\
Analfabeto & 39 & 29 & 10 \\
Ensino Fundamental Incompleto & 6 & 5 & 1 \\
Ensino Fundamental Completo & 1 & 1 & -- \\
Ensino Médio Incompleto & 9 & 8 & 1 \\
Ensino Médio Completo & 1 & -- & 1 \\
Ensino Superior Incompleto & 2 & 2 & -- \\
Ensino Superior Completo & $8,01 \pm$ & $8,28 \pm$ & $6,48 \pm$ \\
\hline Tempo de DM (anos) & 7,18 & 7,7 & 5,05 \\
\hline
\end{tabular}

Os resultados de idade e tempo de DM foram expressos em média \pm Desvio Padrão. ${ }^{*}(p=0,0001)$

Na Tabela 3 é possível identificar a média para os parâmetros clínicos e antropométricos avaliados durante o estudo. Pelo cálculo de IMC pode-se afirmar que $39 \%(n=20)$ dos pacientes classificados como grupo 1 apresentaram-se obesos, pertencentes ao sexo masculino (19,5\%; $n=10)$ e feminino $(19,5 \% ; n=10)$, e $45 \%(n=23)$ com sobrepeso, entre eles $26 \%(n=14)$ homens e $19 \%(n=9)$ mulheres. Já no grupo $2,57 \%(n=8)$ dos pacientes encontram-se obesos, sendo eles do sexo masculino $(37,5 \% ; n=3)$ e feminino $(62,5 \% ; n=5)$ e $36 \%(n=5)$ dos pacientes apresentam-se com sobrepeso, todos do sexo masculino. Quando comparados os valores de IMC para os grupos de alta e baixa adesão, não foram observadas diferenças estatisticamente significativas.

O parâmetro de circunferência do quadril mostrou-se maior no grupo de baixa adesão ao autocuidado $(p=0,043)$, assim como no parâmetro de circunferência abdominal $(p=0,0012)$, sendo que os pacientes que se enquadram nos critérios de baixa adesão possuem este parâmetro aumentado. Dentro de um mesmo grupo, entretanto, não foi possível identificar diferença entre os gêneros. Na relação cintura-quadril foi observada diferença entre os grupos apenas no sexo feminino $(p=0,032)$, contudo ao analisar todos os pacientes, sem distinção de grupos, foi possível observar que $30 \%$ dos homens e $41 \%$ das mulheres possuem risco de desenvolver doenças cardiovasculares, pois apresentam RCQ maior que 1,0 e 0,85, respectivamente. Quando observados os grupos individualmente, tem-se que o grupo 1 apresenta mais mulheres (41\%) propensas a desenvolver doenças cardíacas 
Tabela 3 - Média dos parâmetros clínicos e antropométricos dos pacientes participantes da pesquisa

\begin{tabular}{|l|c|c|c|c|}
\hline \multicolumn{1}{|c|}{ Variável } & $\begin{array}{c}\text { Total } \\
(\mathrm{n}=65)\end{array}$ & $\begin{array}{c}\text { Grupo } 1 \\
\text { Alta adesão }(\mathrm{n}=51)\end{array}$ & $\begin{array}{c}\text { Grupo } 2 \\
\text { Baixa adesão }(\mathrm{n}=14)\end{array}$ \\
\hline IMC (Kg/m $\mathbf{)}$ & $31,70 \pm 0,5$ & $28,54 \pm 0,52$ & $30,94 \pm 1,20$ & $0,04^{*}$ \\
\hline Circunferência do Quadril (cm) & $106,0 \pm 8,12$ & $104,9 \pm 7,54$ & $109,9 \pm 9,21$ & $0,043^{*}$ \\
\hline Circunferência Abdominal (cm) & $104,26 \pm 1,28$ & $102,20 \pm 1,38$ & $111,79 \pm 2,31$ & $0,0012^{*}$ \\
\hline RCQ & $0,987 \pm 0,009$ & $0,98 \pm 0,010$ & $1,02 \pm 0,018$ & $0,035^{*}$ \\
\hline Glicose (mg/dL) & $134,4 \pm 45,79$ & $132,0 \pm 45,87$ & $143,1 \pm 46,12$ & 0,1253 \\
\hline Colesterol Total (mg/dL) & $229,8 \pm 59,55$ & $226,4 \pm 63,40$ & $242,3 \pm 42,28^{*}$ & $0,048^{*}$ \\
\hline Colesterol HDL (mg/dL) & $52,95 \pm 22,62$ & $49,59 \pm 19,00$ & $65,21 \pm 30,39^{*}$ & 0,02 \\
\hline LDL (mg/dL) & $132,00 \pm 62,86$ & $137,8 \pm 64,37$ & $109,9 \pm 53,17$ & 0,109 \\
\hline VLDL (mg/dL) & $48,55 \pm 43,99$ & $42,18 \pm 38,01$ & $71,79 \pm 56,85^{*}$ & 0,024 \\
\hline Triglicerídios (mg/dL) & $234,2 \pm 210,7$ & $212,2 \pm 189,5$ & $314,1 \pm 267,8^{*}$ & 0,0067 \\
\hline
\end{tabular}

do que homens (27\%), e no grupo 2 verifica-se uma igualdade entre os sexos, identificando-se que $85 \%$ dos pacientes possuem probabilidade de desenvolver doenças cardiovasculares.

Ainda, de acordo com a Tabela 3, os valores de glicose mostram-se elevados em $60 \%$ dos pacientes entrevistados, ressaltando-se que $46 \%$ pertencem ao grupo de alta adesão ao autocuidado e $14 \%$ ao grupo de baixa adesão ao autocuidado. Os parâmetros bioquímicos em que foram encontradas diferenças estatisticamente significativas entre os grupos de alta e baixa adesão foram Colesterol Total, Colesterol HDL, VLDL e triglicerídeos.

Os resultados foram expressos em média \pm Desvio Padrão. *As diferenças estatisticamente significantes entre os grupos de alta e baixa adesão foram determinadas pelo Teste $\mathrm{t}$ de Student; $\mathrm{HDL}=$ lipoproteína de alta densidade; $L D L=$ lipoproteína de baixa densidade; VLDL= lipoproteínas de densidade muito baixa; RCQ = Relação Cintura Quadril.

\section{DISCUSSÃO}

O DM é um problema de saúde pública em todo o mundo e vem requerendo atenção pelo alto índice de mortalidade e pela brusca diminuição da qualidade de vida dos pacientes (GALVIN; NAVARRO; GREATTI, 2014). O QAD demonstra que $78 \%$ dos pacientes realizam o autocuidado, porém $61 \%$ obtiveram baixa adesão à alimentação saudável e à prática de exercícios físicos. Esta baixa adesão pode ser justificada pela grande restrição alimentar e alto custo dos alimentos para estes pacientes; já a prática de exercício físico pode não ocorrer pela falta de ânimo, desconforto, falta de tempo, hiperglicemia, falta de orientação médica (GOMIDES et al., 2013).
Apesar da importância da automonitorização da glicemia, apena $9 \%$ dos pacientes o fazem, pois declaram possuir dificuldades para realizar a atividade, necessitando irem até a Unidade Básica de Saúde para avaliação da glicose capilar (VERAS et al., 2014). A conscientização dos pacientes no cuidado com os pés mostra-se importante para diagnosticar problemas precoces, uma vez que está ligado a uma das principais complicações do DM (NETA; SILVA; SILVA, 2015).

Os pacientes apresentam alta adesão ao tratamento medicamentoso (86\%). Este resultado assemelha-se aos obtidos no estudo de Santos et al. (2014), entretanto as Diretrizes da Sociedade Brasileira de Diabetes (OLIVEIRA; MONTENEGRO JUNIOR; VENCIO, 2017) enfatizam que a alta adesão à alimentação saudável, à prática de exercício físico, cuidado com os pés e a automonitorização da glicemia, ou seja, o tratamento não medicamentoso, é quase duas vezes mais efetivo que qualquer tratamento utilizando medicamentos. O tabagismo vem sendo ligado ao risco de ocorrência de morbimortalidade dos pacientes com DM, tendo o estudo este lado positivo, apenas $15 \%$ dos pacientes já utilizaram tabaco.

A idade é grande influenciadora do autocuidado: pessoas com maior faixa etária podem apresentar dificuldades com as recomendações médicas, justificando-se então a não adesão ao autocuidado nessa faixa etária. $O$ gênero não é um fator limitante para o estudo, pois a diferença entre eles é mínima, contudo a amostra é composta por mais homens do que mulheres, porém no grupo de baixa adesão a proporção homens/mulheres é maior. Tanqueiro (2013 apud BAl; CHIOU; CHANG, 2009) argumenta que o gênero é um fator limitante para o autocuidado, deixando claro que o gênero feminino pratica maior autocuidado, devido à função que exerce no seio familiar. 
O baixo nível de escolaridade é uma variável importante quando se fala em autocuidado. Segundo Neta, Silva e Silva (2015), pessoas com menor nível de escolaridade podem apresentar dificuldades de compreender as recomendações terapêuticas dadas pelos profissionais de saúde. Já segundo Boas et al. (2011), a única variável que causou impacto no autocuidado foi a escolaridade, sugerindo que quanto menor a escolaridade, menor a adesão à dieta e ao exercício físico. No presente estudo, entretanto, não foram observadas diferenças significativas quanto ao grau de escolaridade entre pacientes com baixa e alta adesão.

Valores aumentados de IMC, circunferência abdominal e de quadril estão ligados também à adesão aos exercícios físicos evidenciada em ambos os grupos, consequentemente alterando estes parâmetros. Pode-se observar diferenças estatisticamente significativas nos parâmetros CA e CQ, com estas revelando-se maiores no grupo de baixa adesão ao autocuidado, constituído por pacientes que têm menor hábito de praticar exercícios físicos. Segundo Santos et al. (2014), estes índices alterados contribuem para o excesso de peso, adiposidade visceral e altos índices glicêmicos.

Os valores de IMC obtidos pelo presente estudo demonstram que a maioria dos pacientes analisados encontra-se na classificação de sobrepeso e obesidade, além disso nota-se que o grupo de baixa adesão ao autocuidado possui os valores de IMC maiores. É importante destacar que valores de IMC alterados podem estar relacionados aos valores de glicemia em jejum também alterados, em ambos os grupos, pois o excesso de peso pode resultar em resistência à insulina, desta forma o IMC elevado pode contribuir para hiperglicemia, podendo ser uma variável importante para o aparecimento de complicações do diabetes (SANTOS et al., 2014).

Neste contexto, pode-se observar que o grupo com baixa adesão ao autocuidado possui níveis de glicose mais elevados do que o grupo de alta adesão, contudo não foram observadas diferenças significativas, é importante salientar que os valores também ambos os grupos estão acima dos índices preconizados pela Sociedade Brasileira de Diabetes aumentando as chances de complicações micro e macro vasculares (OLIVEIRA; MONTENEGRO JUNIOR; VENCIO, 2017)

Valores mais elevados ainda foram encontrados em estudo realizado por Santos et al. (2014), utilizando o mesmo instrumento de coleta, em que o grupo que possui baixa adesão ao autocuidado possui glicemia de jejum igual a $194,58 \mathrm{mg} / \mathrm{dL}(\mathrm{DP}=82,03)$ e os $\mathrm{pa}$ cientes pertencentes ao grupo de alta adesão ao auto- cuidado apresentam os valores de glicemia de jejum $154,16 \mathrm{mg} / \mathrm{dL}(\mathrm{DP}=65,66)$. Estes dados demonstram a importância da conscientização e aconselhamento dos pacientes no cuidado com a ingestão de alimentos, tomada de medicação e todas as outras variáveis que podem influenciar no aumento da glicemia.

No presente estudo, o RCQ de ambos os gêneros e grupos encontram-se alterados, porém as mulheres do grupo de baixa adesão são as que possuem este índice mais elevado. Fiani, Monteiro e Foss (2009) observaram que existe associação entre o aumento desta variável com a glicemia e massa corporal, pois a gordura abdominal é um importante fator para desordem metabólica, além de a gordura visceral estar também associada a muitas dislipidemias.

Por fim, observou-se que os valores de colesterol total e triglicerídeos dos pacientes analisados no estudo encontram-se alterados, sendo possível observar diferenças estatisticamente significativas entre os grupos, revelando que os pacientes com baixa adesão ao autocuidado possuem os índices lipêmicos maiores.

Os valores de LDL encontram-se alterados apenas no grupo de alta adesão ao autocuidado, assim com os níveis de HDL são maiores no grupo de baixa adesão ao autocuidado. O exercício físico é o principal fator envolvido para o aumento deste tipo de colesterol (FRAGA et al., 2017). Neste estudo, a falta de adesão a este critério pode estar altamente ligada aos baixos níveis de HDL. Segundo as Diretrizes da Sociedade Brasileira de Diabetes (OLIVEIRA; MONTENEGRO JUNIOR; VENCIO, 2017), pacientes com DM2 são frequentemente portadores de uma série de fatores de risco para doenças aterotrombóticas, entre os quais a dislipidemia provavelmente exerça o papel mais importante.

O nível de colesterol total elevado vem sendo um grande fator de risco para o desenvolvimento de doenças cardiovasculares, este risco pode ser aumentado com a ampliação dos níveis de LDL e triglicerídeos e diminuição dos níveis de HDL (GRILO; GORI$\mathrm{NI}$ 2007). As dislipidemias exercem efeito tóxico nas células beta do pâncreas e quando associadas com a hiperglicemia podem acarretar aumento do potencial da ocorrência de doenças cardíacas (OLIVEIRA; MONTENEGRO JUNIOR; VENCIO, 2017).

É importante ressaltar que o QAD foi realizado refletindo apenas a última semana de autocuidado dos pacientes, portanto não se sabe há quanto tempo eles praticam estes cuidados. Como estratégia para aumentar a adesão às atividades de autocuidado po- 
dem ser utilizados como ferramentas grupos de estudos mensais para abordagem de assuntos relevantes quando se trata de DM.

\section{CONSIDERAÇÕES FINAIS}

Com o presente estudo é possível confirmar que o autocuidado pode influenciar os parâmetros bioquímicos e antropométricos dos pacientes diabéticos, notando-se que os principais influenciadores são a falta de regras na alimentação e de atividade física, os quais, dentro do autocuidado, demonstram-se os fatores mais fortes para o aumento da glicemia e desenvolvimento de comorbidades.

O grande obstáculo para os pacientes diabéticos é justamente a conscientização da necessidade de praticar o autocuidado, especialmente aqueles relacionados à alimentação, atividade e exercício físico. Por estes motivos, os profissionais de saúde são importantes para promover o estímulo, motivação e além de tudo ajudar os pacientes a desenvolver habilidades para o seu próprio cuidado, levando em conta as limitações e comorbidades de cada paciente.

\section{AGRADECIMENTOS}

A autora agradece à Universidade Regional Integrada do Alto Uruguai e das Missões - URI Campus Santo Ângelo e ao município de Santo Ângelo. Agradecimentos também à professora orientadora doutora Karine Santos De Bona Libardoni por toda a competência e paciência ao longo do trabalho.

\section{REFERÊNCIAS}

ASSOCIAÇÃO BRASILEIRA PARA O ESTUDO DA OBESIDADE E DA SÍNDROME METABÓLICA (Abeso). Diretrizes brasileiras de obesidade. 4. ed. São Paulo, SP: Abeso, 2016.

BOAS, L. C. G. et al. Adesão à dieta e ao exercício físico das pessoas com diabetes mellitus. Texto Contexto Enfermagem, São Paulo, v. 2, n. 20, p. 272-279, jun. 2011.

BRASIL. Ministério da Saúde. Secretaria de Atenção Básica. Departamento de Atenção Básica. Estratégia para o cuidado da pessoa com doença crônica: diabetes mellitus. Brasília: Ministério da Saúde, 2013.
FIANI, C. R. V.; MONTEIRO, L. Z.; FOSS, M. C. Avaliação da relação cintura/quadril com glicemia e massa corpórea em Diabéticos Tipo 2. Revista EpeQ/Fafibe, Bebedouro, v. 1, n. 1, p. 29-35, 2009.

FRAGA, A. S. et al. Efeito do exercício sobre os níveis de HDL-C: uma revisão sistemática de metanálise. Revista Brasileira Medicina e Esporte, Bahia, v. 23, n. 6, p. 488-494, 2017.

GALVIN, E. A.; NAVARRO, F.; GREATTI, V. R. A importância da prática do exercício físico para portadores de Diabetes Mellitus: uma revisão crítica. Salusvita, Bauru, v. 33, n. 2, p. 209-222, 2014.

GOMIDES, D. S. et al. Autocuidado das pessoas com Diabetes Mellitus que possuem complicações em membros inferiores. Acta Paul Enferm, Ribeirão Preto, v. 26, n. 3, p. 289293, jan. 2013.

GRILO, M. F. F.; GORINI, M. I. P. C. Caracterização de pessoas com Diabetes Mellitus tipo 2. Revista Brasileira de Enfermagem, Brasília, v. 60, n. 1, p. 49-54, 2007.

MICHELS, M. J. et al. Questionário de atividades de autocuidado com o diabetes: tradução, adaptação e avaliação das propriedades psicométricas. Arquivos Brasileiros de Endocrinologia e Metabologia, Florianópolis, v. 7, n. 54, p. 644651, jan. 2010.

NETA, D. S. R.; SILVA, A. R. V.; SILVA, G. R. F. Adesão das pessoas com diabetes mellitus ao autocuidado com os pés. Revista Brasileira de Enfermagem, Teresina, v. 1, n. 68, p. 111-116, fev. 2015.

OLIVEIRA, José Egídio Paulo de; MONTENEGRO JUNIOR, Renan Magalhães; VENCIO, Sérgio (Org.). Diretrizes da Sociedade Brasileira de Diabetes 2017-2018. São Paulo: Ed. Clannad, 2017.

SANTOS, G. F. P. et al. A adesão ao autocuidado influência parâmetros bioquímicos e antropométricos de pacientes diabéticos tipo 2 atendidos no programa hiperdia do município de Juiz de Fora, Minas Gerais, Brasil. Nutr. Clín. Diet. Hosp., Minas Gerais, v. 3, n. 34, p. 10-19, jan. 2014.

TANQUEIRO, M. T. O. S. A gestão do autocuidado nos idosos com diabetes: revisão sistemática da literatura. Revista de Enfermagem Referência, Coimbra, v. 3, n. 9, p. 151-160, mar. 2013.

VERAS, V. S. et al. Autocuidado de pacientes inseridos em um programa de automonitorização da glicemia capilar no domicílio. Revista Gaúcha de Enfermagem, Ribeirão Preto, v. 4, n. 35, p. 42-48, dez. 2014.

WORD HEALTH ORGANIZATION (WHO). Obesity: preventing and managing the global epidemic. Geneva: Program of Nutrition, Family and Reproductive Health, 1998. (Technical Report Series 894). 